\title{
Medical Image Fusion based on Pulse Coupled Neural Network Combining with Compressive Sensing
}

\author{
AiliWang ${ }^{1}$, Jiaying Zhao ${ }^{1}$, Shiyu Dai ${ }^{1}$, Yuji Iwahori ${ }^{2}$ and Yangyang Zhao \\ ${ }^{1}$ Higher Education Key Lab for Measuring \& Control Technology and \\ Instrumentations of Heilongjiang, Harbin, China \\ ${ }^{2}$ Dept. of Computer Science, Chubu University, Japan \\ aili925@hrbust.edu.cn
}

\begin{abstract}
Image fusion is an important branch of information fusion, widely used in various fields, especially in medical field. So increasing the quality and efficiency of medical image fusion has great significance. Combining the advantages of pulse coupled neural networks with Compressive Sensing; this paper puts forward a novel image fusion method in NSCT transform domain. First, NSCT transform is applied to the source images, and the coefficients in low frequency coefficient are fused by mean rules. For high frequency coefficient, CS is applied and PCNN. Finally, inverse NSCT is applied to get the reconstructed image. The experimental results show that the fusion algorithm proposed in this paper in the performance and integration efficiency has better fusion results.
\end{abstract}

Keywords: image fusion, PCNN, CS, NSCT

\section{Introduction}

Image fusion refers to the different levels of integrated information from a plurality of images; these images may be obtained from the same type of image sensor, can also be obtained from different types of sensors, and by some of the fusion obtained containing more information the new image method. Fused image should be more suitable for the subsequent processing of the human visual perception or computer. Compared with the original image, new image information is more comprehensive, accurate and stable. Therefore, image fusion has been widely used in satellite remote sensing, computer vision, medical imaging and military applications $[1,2]$.

As a direct fusion of image pixels, fused image has obvious splicing signs, so the current image fusion is to be transformed in the transform domain. NSCT (non-subsampled contourlet transform) has the following advantages: multi-scale, local time-frequency characteristics, translation invariance and the image may be sparse representation and it is widely applied to image fusion [3,4]. Since the image data becomes larger after NSCT transform, lower fusion efficiency. Compressive sensing (CS) theory proposed by Candes and Donoho just can solve this problem [9], and achieve very good results.

In fact, humans are often sensitive to image's edges, directional features, etc. So, a pure use of single pixel for image fusion is not enough, it is necessary to consider the global features. PCNN is a novel biological neural network based on the experimental observations of synchronous pulse bursts in cat and monkey visual cortex, which is developed by Eckhorn in 1990[5] . It is characterized by the global coupling and pulse synchronization of neurons. Taking into account the overall characteristics, these characteristics benefit image fusion which makes use of local image information. PCNN has been successfully used in image fusion. PCNN first applied to image fusion is by 
Broussard in order to improve recognition accuracy. Li Wei proposed that wavelet decomposition combining with PCNN method for image fusion [6]. However, due this method used wavelet transform to the image processing, and therefore fused image had edge shift phenomenon. Qu XiaoBo [7] and Zhang Ke [8], et al., proposed image fusion method that combined NSCT transform and PCNN, and achieved good fusion results.

Therefore, this paper proposes a novel image fusion algorithm based on PCNN. First NSCT transform is applied to source images. In the NSCT transform domain using the mean rule for fusion of low frequency coefficients. Then CS is applied for high frequency coefficients for dimensionality reduction, and applies PCNN to fuse high frequency information [10, 11]. Then CS reconstruction and NSCT inverse transform is used to obtain fused image. Experimental results show that the method proposed in this paper can achieve better fusion results than the method based on the maximum energy of neighborhood area in literature [12].

\section{Principles of CS}

CS theory asserts that one can recover certain signals and images from far fewer samples or measurements than traditional methods use. The overall framework of compressive sensing theory is as follows.

Considering a finite one-dimensional discrete-time signal $\mathrm{x}$, which we can expend in an orthonormal basis as follows:

$$
\theta=\Psi^{T} X
$$

Where: $X$ is a finite $\mathrm{n}$-dimensional discrete-time signal, $X \in \mathrm{R}^{\mathrm{n} \times \mathrm{n}} ; \Psi$ is an orthonormal basis, $\Psi \in \mathrm{R}^{\mathrm{n} \times \mathrm{n}} ; \theta$ is the coefficients vector of $\mathrm{x}$ on the orthonormal basis $\Psi, \theta \in \mathrm{R}^{\mathrm{n} \times \mathrm{n}} ; \mathrm{n}$ is the signal dimension. The vector $\theta$ is a sparse vector when all but a few of its entries are zero. If there are only k nonzero entries in the $\theta$, we will call the signal $X$ a k-sparse signal on the basis $\Psi$.

On this basis, if we can construct a measurement matrix $\Phi$, we can get (2).

$$
Y=\Phi \theta=\Phi \Psi^{T} X
$$

Signal reconstruction, this paper uses the Orthogonal Matching Pursuit (OMP) as reconstruction algorithm. Greedy iterative method is used to select $\Phi$ column , then make the column selected in each iteration with the maximum degree of redundancy in the current vector related subtracted from the relevant part of the measurement vector and iteratively, know the number of iterations to achieve the sparsity $\mathrm{M}$, this time to stop iteration.

Figure 1 is the theoretical framework of compressed sensing.

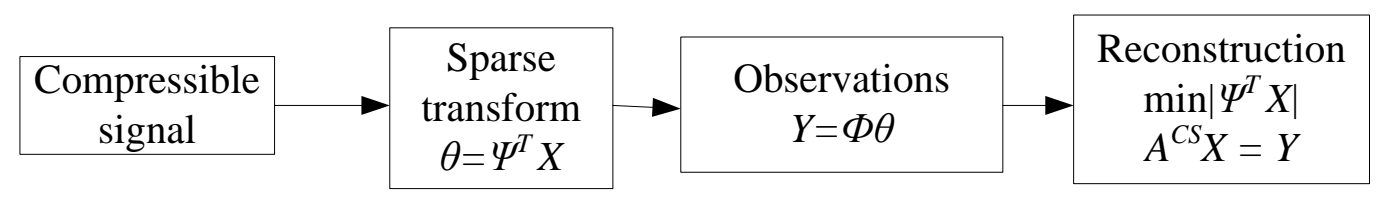

Figure 1. Framework of Compressive Sensing Theory

\section{Basic Theory of PCNN}

The PCNN is derived from the phenomena of synchronous pulse burst in mammals' visual cortex. The PCNN model was first proposed by Eckhorn in 1989 in [6], but it's too complex to be applied to, many improvements were made by different researchers, the neuron model proposed by Ranganath H S is most widely used. The structural model is shown in Figure 2.The model contains three parts: the input part, the linking part and the pulse generator part. The input part consists of the feeding input $F$ and linking input $L$ 
channels. $F$ is the primary input $\mathrm{S}$ from the neuron's receptive field and $L$ is the secondary input of lateral connections with the neighboring neurons. The internal activity $U$ is generated by the modulation of $F$ and $L$. The pulse generator part includes a pulse generator, a threshold adjuster, and a comparison organ. When the internal activity $U$ is larger than the dynamic threshold, the neuron produces a pulse.

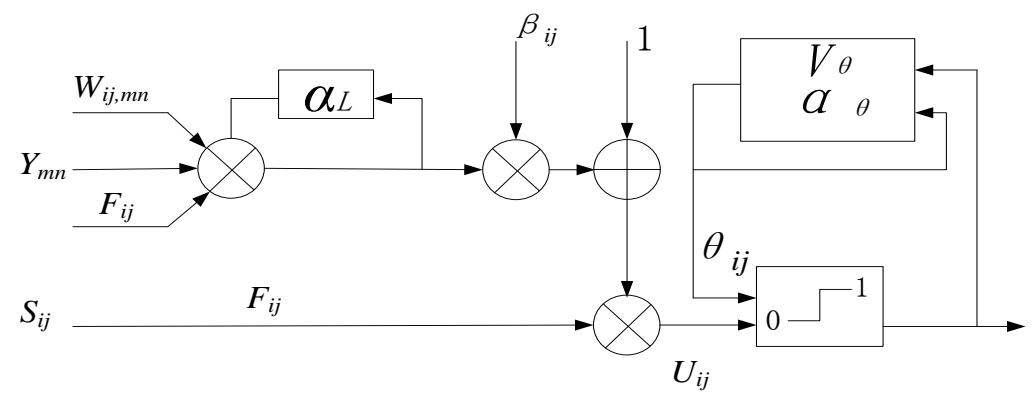

Figure 2. Neuron's structure

The PCNN model for image processing is made up of a 2-D monolayer neuron network. The number of neurons equals to the number of pixels in an image, each pixel has its corresponding neuron. Figure 3 is the structure of PCNN network.

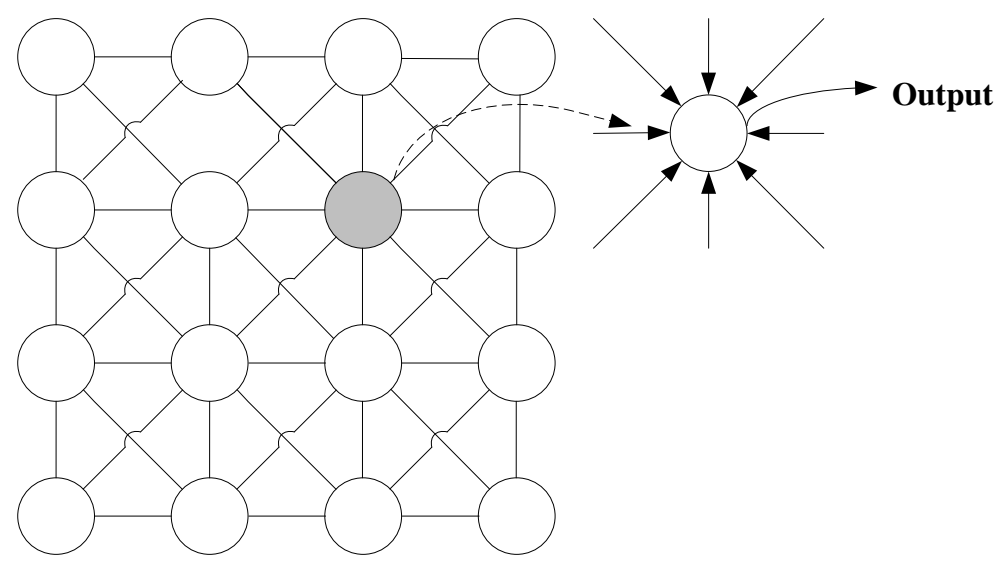

Figure 3. Structure of PCNN

The mathematical description of the PCNN model we apply is as follow [5]:

$$
\left\{\begin{array}{c}
F_{i j}^{k}(n)=I_{i j}^{k} \\
L_{i j}^{k}(n)=e^{-\alpha_{L}} L_{i j}^{k}(n-1)+V_{L} \sum_{m, n} W_{i j, m n} Y_{m n}(n-1) \\
U_{i j}^{k}(n)=F_{i j}^{k}(n) *\left(1+\beta L_{i j}^{k}(n)\right) \\
\theta_{i j}^{k}(n)=e^{-\alpha_{\theta}} \theta_{i j}^{k}(n-1)+V_{\theta} Y_{i j}^{k}(n-1) \\
Y_{i j}^{k}(n)= \begin{cases}1, & \text { 当 } U_{i j}^{k}(n)>\theta_{i j}^{k}(n) \text { 时 } \\
0, & \text { 当 } U_{i j}^{k}(n) \leq \theta_{i j}^{k}(n) \text { 时 }\end{cases}
\end{array}\right.
$$

$\mathrm{S}$ is the original stimulus from the input image: the grayscale of pixels; $n=1,2,3 \ldots, \mathrm{N}$ is the iteration number; $(i, j)$ stands for the position of the neutron in the network and $(m, n)$ is that of its neighboring neurons; $\alpha_{L}, \alpha_{\theta}$ are the attenuation time constant of $L, \theta$, 
respectively; $V_{L}$ and $V_{\theta}$ amplitude gain of $L$ and $\theta ; \mathrm{W}$ represent the synaptic weight matrix of $L ; \beta$ is the linking strength constant; $Y_{i j}(1$ or 0$)$ represents the firing state of the ijth neuron.

Each neuron in PCNN may generate a pulse when stimulus is larger than its dynamic threshold, then the pulse captures its neighboring neurons which have the same characteristics to fire synchronously. That is the capture character and synchronous pulse activity character of PCNN. During the iterations, each neuron has its firing period and dynamic threshold attenuating respectively, so the PCNN produces a binary firing image after each iteration. The number of firing neurons in each iteration is computed and stored in an array: $\mathrm{G}(\mathrm{n})=\sum_{\mathrm{m}, \mathrm{n}=0}^{\mathrm{m}=\mathrm{i}=\mathrm{j}} \mathrm{Y}_{\mathrm{mn}}(\mathrm{n})$. That is the time series stated by Johnson, the earliest way to extract feature from PCNN outputs.

\section{Medical Image Fusion based on Compressive Sensing and Pulse Coupled Neural Network}

Source images are image A and image B, the result of the fusion is image F. Apply NSCT to image A and B respectively to get transform coefficients in low frequency subband and high frequency subband. Different fusion rules are used for low and high frequency coefficients. Figure 4 is the block diagram of fused rule.

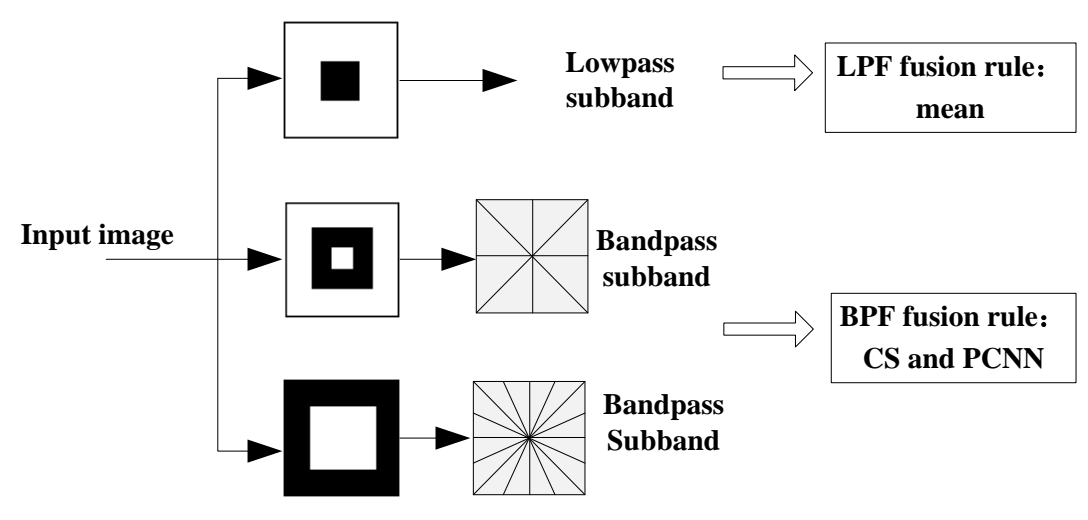

Figure 4. Fusion Rule in NSCT Domain

The following sections introduce the low-frequency and high-frequency region fusion methods.

\subsection{Fusion of Low Frequency Subband Coefficients}

The low frequency part of the overall trend information includes image information, and the overall intensity of the image. For the low-frequency part of this direct integration using the method mean calculation is simple, easy to implement and fusion works well. The pixel value is set $F_{\text {low }}(i, j)$ for the fused image at $(\mathrm{x}, \mathrm{y}), L A_{K}(i, j)$ is the pixel value at $(\mathrm{i}, \mathrm{j})$ of the K level NSCT decomposition source image $\mathrm{A}, L B_{K}(i, j)$ is the pixel value at $(\mathrm{i}, \mathrm{j})$ of the $\mathrm{K}$ level NSCT decomposition source image $\mathrm{B}$.

$$
F_{\text {low }}(x, y)=\frac{L A_{i, j}(x, y)+L B_{i, j}(x, y)}{2}
$$

\subsection{Fusion of High Frequency Subband Coefficients}


Due to the high frequency part reflects the details of the image, and each pixel are not independent of each other, with other surrounding pixels have the necessary contacts. Therefore, should choose a fusion method can be considered global for the high frequency part. PCNN on such a method which can extract useful information from complex background, with a sync pulse distribution and global coupling and other characteristics, its signal form and handling mechanism are more in line with physiological basis of human visual nervous system, so in this paper, we use PCNN fusion high-frequency coefficients. Also taking into account the image is out NSCT, increase the amount of data, increasing the computational complexity, reducing fusion efficiency. Therefore, we first CS transform before PCNN fusion A.

In formula (4), the attenuation coefficient and amplitude gain of $\mathrm{L}$ is $\alpha_{L}=1, \mathrm{~V}_{\mathrm{L}}=$ 0.2 , internal connection matrix of neuron is $W_{i j, m n}=\frac{1}{(\mathrm{i}-\mathrm{m})^{2}+(\mathrm{j}-\mathrm{n})^{2}}$; in formula (6), the attenuation coefficient and amplitude gain of $\theta$ is $\alpha_{\theta}=2, \mathrm{~V}_{\theta}=20$, in formula (5), the connection strength is $\beta=\sum_{\mathrm{i}, \mathrm{j} \in \mathrm{M}}\left(\mathrm{CA}_{\mathrm{ij}}(\mathrm{x}, \mathrm{y})-\mathrm{CA}_{\mathrm{ij}}(\mathrm{x}+1, \mathrm{y})\right)^{2}+\left(\mathrm{CA}_{\mathrm{ij}}(\mathrm{x}, \mathrm{y})-\mathrm{CA}_{\mathrm{ij}}(\mathrm{x}, \mathrm{y}+\right.$ 1) $)^{2}$.

For the fusion result:

$$
\begin{cases}C F_{\text {high }}(x, y)=C A_{i j}(x, y) & \text { when } Y_{i j}^{k}=1 \\ C F_{\text {high }}(x, y)=C B_{i j}(x, y) & \text { when } Y_{i j}^{k}=0\end{cases}
$$

Where, $\mathrm{CF}_{\mathrm{high}}(\mathrm{x}, \mathrm{y})$ is observations at $(\mathrm{x}, \mathrm{y})$ of fused image, $\mathrm{CA}_{\mathrm{ij}}(\mathrm{x}, \mathrm{y}), \mathrm{CB}_{\mathrm{ij}}(\mathrm{x}, \mathrm{y})$ are observations at $(\mathrm{x}, \mathrm{y})$ of source image A and B in NSCT domain.

The following is specific implementation of the proposed image fusion algorithm.

Input: medical image A (CT) and image B (MR).

Step 1: Apply NSCT to image A and B respectively to get transform coefficients in low frequency subband $L A_{i, j}(x, y) 、 L B_{i, j}(x, y)$ and high frequency subband $H A_{i, j}(x, y) 、 H B_{i, j}(x, y)$.

Step 2: We use method of the formula (8) in low frequency subband of image A and B, obtained fused Low frequency coefficient $F_{\text {low }}$.

Step 3: Apply CS to $H A_{i, j}(x, y) 、 H B_{i, j}(x, y)$, obtain $C A_{i, j}(x, y) 、 C B_{i, j}(x, y)$

Step 4: Fusion $C A_{i, j}(x, y) 、 C B_{i, j}(x, y)$ by PCNN method, obtain $C F_{\text {high }}$.

Step 5: Apply OMP algorithm to perform CS reconstruction for $C F_{\text {high }}$, getting the coefficients in high frequency subband, obtain $F_{\text {high }}$.

Step 6: Use inverse NSCT (INSCT) to the fused coefficients both in low frequency subband $F_{\text {low }}$ and high frequency subband $F_{\text {high }}$ to get the final image F.

Output: fused medical image.

\section{Experiments and Results Analysis}

Experiments of medical image fusion are run based on MATLAB7.0. For the experimental results we used the following indicators to determine the assessment, including peak signal to noise ratio (PSNR), cross-entropy (CERF), mutual information (MI), edge information (QABF), entropy $(\mathrm{H})$, spatial frequency information(SF), average gradient (AVE). Among them, the values of PSNR, MI, QABF, H, SF, AVE are bigger, and the CERF is smaller, the fusion result is better.

Medical imaging has become an integral part of modern medicine, its application throughout the clinical work, not only widely used in disease diagnosis, but also plays an important role in surgery and radiation therapy program design, program implementation and efficacy assessments. E.g. MR applicable to tumor tissue contour description, CT images can be accurately calculated tumor dose, and therefore often need to be in the course of radiotherapy either fusion diagnosis, and therefore selected CT images and MR images of $256 \times 256$ to experiment in this paper. 
Table 1 is the results by the method in this paper (CS-PCNN-5), among them NSCT decomposition level which is two layers four directions, five times the number of iterations. At the same time compared method that directly PCNN integration after NSCT by Zhang Ke, et al., (PCNN-5) and regional energy integration method (CS_NE_MAX) form literature [12]. The following is specific experimental data

Table 1. Fusion Results of Different Methods

\begin{tabular}{c|c|c|c|c|c|c|c|c}
\hline \multicolumn{1}{c|}{ Method } & PSNR & CERF & MI & QABF & H & SF & AVE & $\begin{array}{c}\text { TIM } \\
\text { E }\end{array}$ \\
\hline CS-NE-MAX & 32.753 & 2.222 & 14.747 & 0.677 & 5.954 & 8.078 & 0.0210 & 101 \\
\hline PCNN-5 & 32.463 & 2.768 & 14.689 & 0.689 & 5.9765 & 9.1086 & 0.0247 & $\begin{array}{c}298.0 \\
1\end{array}$ \\
\hline CS-PCNN-5 & 31.291 & 2.147 & 15.088 & 0.772 & 5.988 & 9.111 & 0.0251 & 172 \\
\hline
\end{tabular}

The experimental results in Table 1 show that for the objective evaluation, in addition to PSNR and TIME, other indicators are better than CS-NE-MAX methods. This is due to PCNN has a global approach and can consider the relationship between the overall pixels, but the CS-NE-MAX method only considers the relationship between the pixel within the energy of region, so the method in this paper better than CS-NEMAX method. Since the method in paper on evaluation much better than CS-NEMAX method, spending extra time for 70s cannot be considered. Comparison with known methods PCNN-5, the method of this paper is not only in the objective evaluation and time spent on integration methods are superior PCNN-5's, because the method of this paper CS transformation NSCT coefficients, reducing the amount of data while reducing sparsity between pixels, improve the correlation between each other, therefore, the method in this paper has certain advantages.

Figure 5 give the fused images by different fusion methods. Figure 5 (a) is CT image,(b) is MR image, (c), (d), (e) are the fused result image by CS-NE-MAX, PCNN-5 and CS-PCNN-5. Through fused images can also be aware that these method fusion image NSCT decomposition 2 layer 4 directions obtained at the edge of the area and internal texture information are better than several other methods.

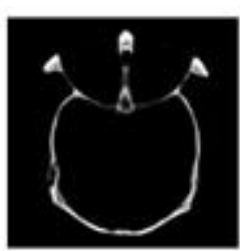

(a) $\mathrm{CT}$

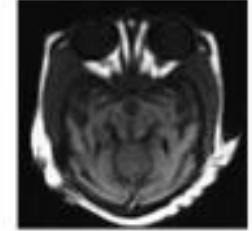

(b) MR

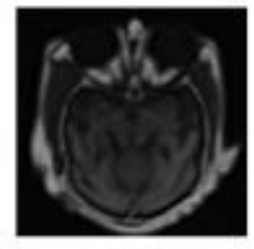

(c) CS-NE-MAX

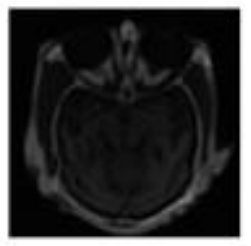

(d) PCNN-5

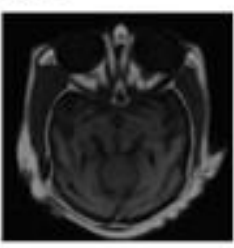

(e) $\mathrm{CS}-\mathrm{PCNN}-5$

Figure 5. Fused Images by Different Fusion Methods 
Based on the experimental results above, this article has conducted experiments to verify the number of iterations of the impact of fusion results, among them NSCT decomposition which are two layers four directions. Table 2 is the result of image fusion, PCNN iterations which were 20 times (CS -PCNN-20), 10 times (CS -PCNN-10), 5 times (CS-PCNN-5) and CS without conversion (PCNN-5).

Table 2. Comparison of Different Iterations of PCNN's Effect on Fusion Results

\begin{tabular}{l|c|c|c|c|c|c|c|c}
\hline Methods & PSNR & CERF & MI & QABF & H & SF & AVE & TIME \\
\hline $\begin{array}{l}\text { CS-PCNN } \\
-20\end{array}$ & 31.291 & 2.148 & 15.0688 & $\begin{array}{c}0.772 \\
2\end{array}$ & $\begin{array}{c}5.988 \\
6\end{array}$ & $\begin{array}{c}9.110 \\
9\end{array}$ & $\begin{array}{c}0.025 \\
3\end{array}$ & 644.562 \\
\hline $\begin{array}{l}\text { CS-PCNN } \\
-10\end{array}$ & 31.2898 & 2.152 & 15.1077 & $\begin{array}{c}0.772 \\
5\end{array}$ & $\begin{array}{c}5.988 \\
5\end{array}$ & $\begin{array}{c}9.109 \\
9\end{array}$ & $\begin{array}{c}0.025 \\
3\end{array}$ & 332.015 \\
\hline $\begin{array}{l}\text { CS-PCNN } \\
-5\end{array}$ & 31.2913 & 2.147 & 15.0882 & $\begin{array}{c}0.772 \\
4\end{array}$ & 5.988 & $\begin{array}{c}9.111 \\
3\end{array}$ & $\begin{array}{c}0.025 \\
1\end{array}$ & 172.717 \\
\hline
\end{tabular}

From Table 2, it can be seen the iteration number's increase did not make the fusion results to be better, in contrast, when the number of iterations increase, the time needed to increase the integration process doubled, affect the efficiency of fusion. Because high-frequency coefficients obtained by NSCT decomposition is sparse, the correlation reduces between pixels. When the number of iterations increasing, the correlation between pixels becomes large, the fusion effect is not better, however, due to the increased number of iterations, the calculation amount is increased, so that the relative increase in fusion time.

\section{Conclusion}

PCNN considers global characteristics of image and the characteristics of neural networks for each pixel. Therefore, this paper proposes a novel image fusion method based on PCNN and CS theory by combining the advantages of PCNN with advantages of CS for dimension reduction. And experiment results show that the method proposed in this paper in terms of subjective visual and objectively indicators have better results for the following recognition and detection applications. Meanwhile, experiments were carried out in order to study the NSCT decomposition level iterations and PCNN iterations' impact on experimental results. It can be concluded the proposed method that convert the image into NSCT domain with 2-layer 4-direction, reduce dimension by CS, and finally process five iterations of PCNN for image fusion can give optimal fusion results.

\section{References}

[1] Y. C. Lin and T. H. Chen, "Infrared and Vsible Image Fusion Method Based on Wavelet Transform", 2013 International Conference on Advanced Computer Science and Electronics Information, (2013), pp. 632-635.

[2] X. Wen and C. Li, "Feature Level Image Fusion for SAR and Optical Images", 2011 4th IEEE International Conference on Computer Science and Information Technology, (2011), pp. 462-466.

[3] M. N. Do and M. Vetterli, "The contourlet transform: an efficient directional multiresoulution image representation", IEEE Transactions on Image Processing, vol. 14, no. 12, (2005), pp. 2091-2106.

[4] A. L. Cunha, J. P. Zhou and M. N. DO, "The nonsubsampled contourlet transform theory design and applications", IEEE Trans. on Image Processing, vol. 15, no. 10, (2006), pp. 3089-3101.

[5] E. Candes, J. Romberg and T. Tao, "Robust Uncertainty Principles: Exact Signal Reconstruction from Highly Incomplete Frequency Information”, IEEE Trans Information Theory, vol. 52, no. 2, (2006), pp. 489-509. 
[6] H. Chen and J. Liu, “A Distributed Compressed Sensing for Images Based on Block Measurements Data Fusion", Proceedings of Conference on Computer Science and Software Engineering, CSSE, (2012), pp. 134-140.

[7] Z. Pai, H. Chun-Hai and Z. Hai-Feng, "A Novel Image Fusion Algorithm Based On An Improved Compressed Sensing”, Proceedings of 2012 IEEE 11th International Conference on Signal Processing ICSP, (2012), pp. 672-676.

[8] R. Eckhorn, H. J. Reitboeck, M. Arndt, et al., "Feature linking via synchronization among distributed assemblies: Simulations of results from cat visual cortex", Neural Comp, vol. 2, no. 3, (1990), pp. 293-307.

[9] W. Li and Z. Xue-Feng, "A new algorithm of multi-modality medical medical image based on pulse coupled neural networks", Advances in Natural Computation: First International Conference ICNC Berlin: Springer, (2005), pp. 995-1001.

[10] Q. Xiao-Bo, Y. Jing-Wen, et al., "Image Fusion Algorithm Based on Spatial Frequency-Motivated Pulse Coupled Neural Networks in Nonsubsampled Contourlet Transform Domain", ACTA AUTOMATICA SINICA, (2008), pp. 1508-1514.

[11] L. Mei-Li, L. Yan-Jun, Z. Ke and L. Yan-Jun, "Fusion Algorithm of Infrared and Visible Images Based on NSCT and PCNN", Opto-Electronic Engineering, vol. 37, no. 6, (2010), pp. 90-95.

[12] L. Zhenkun and Y. Z. Henming, "Multi focus image fusion based onlocalized Gabor energy", Application Research of Computers, vol. 27, no. 5, (2010), pp. 1944- 1951. 